\title{
RM en el diagnóstico y control evolutivo de la degeneración combinada subaguda. A propósito de un caso
}

\author{
MRI in the diagnostic and evolutive control of subacute \\ combined degeneration. A case report
}

\author{
R. Saiz-Mendiguren ${ }^{1,3}$, R. García-Eulate ${ }^{1}$, A. García Lallana ${ }^{1,4}$, P. Irimia ${ }^{2}$, \\ E.A. Martínez Vila ${ }^{2}$
}

\section{RESUMEN}

El déficit de vitamina B12, consecuencia generalmente de la anemia perniciosa, puede dar lugar a trastornos de la médula espinal, cerebro, nervios ópticos y periféricos. El principal síndrome neurológico es la degeneración combinada subaguda de la médula (DCS), que puede causar alteraciones motoras y/o sensitivas progresivas, inestabilidad e incontinencia, debido a la desmielinización de los cordones posteriores de la médula. La identificación por resonancia magnética (RM) de una hiperintensidad de señal en secuencias potenciadas en $\mathrm{T} 2$ a nivel de los cordones posteriores de la médula cervical y/o dorsal, puede ser de gran utilidad en la orientación diagnóstica del paciente con DCS, sobre todo cuando los síntomas son leves o inespecíficos, y el paciente no tiene alteraciones hematológicas o gastrointestinales. Además, la evolución de la alteración de la señal del cordonal posterior en RM puede ser de utilidad para valorar la eficacia del tratamiento, ya que su normalización se relaciona con la mejoría clínica.

Palabras clave. Degeneración combinada subaguda. Médula espinal. Resonancia magnética. Vitamina B12.

\begin{abstract}
A deficit of vitamin B12, generally resulting from pernicious anaemia, can give rise to disorders of the spinal cord, brain, optic and peripheral nerves. The principal neurological syndrome is subacute combined degeneration of the spinal cord (SCD), which can cause progressive motor and/or sensitive alterations, instability and incontinency, due to the demyelination of the posterior horn of the spinal cord. The identification by magnetic resonance (MR) of signal hyperintensity in T2 weighted sequences at the level of the posterior horns of the spinal and/or cervical cord can be of great use in diagnosising the patient with SCD, above all when the symptoms are mild or nonspecific, and the patient does not have haematological or gastrointestinal alterations. Besides, the evolution of the altered signal of the posterior horns in MR can be of use in evaluating the efficacy of treatment, since their normalization is related to clinical improvement.
\end{abstract}

Key words. Subacute combined degeneration. Spinal cord. Magnetic resonance. Vitamin B12.
1. Servicio de Radiología. Clínica Universidad de Navarra. Pamplona. España

2. Servicio de Neurología. Clínica Universidad de Navarra. Pamplona. España

3. Servicio de Radiología. Clínica Juaneda. Palma de Mallorca. España

4. Servicio de Radiología. Hospital San Juan de Dios. Santurce (Bizkaia). España

Recepción: 20 de febrero de 2012

Aceptación provisional: 28 de febrero de 2012

Aceptación definitiva: 12 de abril de 2012

\section{Correspondencia:}

Ramón Saiz Mendiguren

Servicio de Radiología

Clínica Universidad de Navarra

Avda. Pío XII № 36

31008-Pamplona. España

e-mail: saiz.ramon@gmail.com 


\section{INTRODUCCIÓN}

El déficit de vitamina B12 puede causar alteraciones en la médula espinal, el encéfalo, los nervios ópticos y/o periféricos y dar lugar a síntomas muy variados ${ }^{1,2}$. Los estados carenciales de vitamina B12, se deben habitualmente a defectos en su absorción o metabolismo. En los países desarrollados, la causa más frecuente es la enfermedad autoinmune por autoanticuerpos frente al factor intrínseco (FI) o las células parietales gástricas. El principal síndrome neurológico por déficit de vitamina B12, es la degeneración combinada subaguda de la médula (DCS), y con frecuencia la única afectación neurológica. Las manifestaciones clínicas son variadas, e incluyen parestesias en extremidades, alteraciones de la sensibilidad posicional y vibratoria, inestabilidad, trastornos esfinterianos, debilidad progresiva y paraplejia si no se instaura el tratamiento. Aunque en los pacientes con DCS, el déficit de B12 se suele detectar en base a la frecuente coexistencia de alteraciones hematológicas (anemia megaloblástica, macrocitosis) o gastrointestinales, en ocasiones estos síntomas no están presentes o aparecen en fases avanzadas ${ }^{3}$.

El condicionante más importante de la recuperación neurológica en la DCS, es la rapidez con que se instaure el tratamiento con vitamina B12, por lo que la precocidad en el diagnóstico resulta fundamental. En pacientes con DCS, la resonancia magnética (RM) puede mostrar una hiperseñal en los cordones posteriores de la médula cervico-dorsal en secuencias potenciadas en $\mathrm{T} 2$, que orienta el diagnóstico, sobre todo en las fases iniciales, cuando los síntomas son leves o inespecíficos ${ }^{4}$.

\section{CASO CLÍNICO}

Varón de 75 años, sin antecedentes de interés, que presenta un cuadro de dos meses de evolución y curso lentamente progresivo que el paciente describe como "pinchazos" en las puntas de los dedos, y "acorchamiento", que inicialmente notaba en ambas manos con sensación de pérdida del tacto, pero que, en el último mes, también afecta a ambos pies. En la exploración neurológica, se apreció hipoestesia táctil con distribución en "guante» $\mathrm{y}$ "cal- cetín", apalestesia maleolar e hipopalestesia en crestas tibiales y codos. Los reflejos estaban presentes y eran simétricos. El resto de la exploración neurológica fue normal. La presencia de parestesias, con una alteración de la sensibilidad vibratoria y preservación de reflejos en las exploración neurológica, obliga a considerar que el paciente sufra una lesión medular cervical con afectación de los cordones posteriores. Se solicitó analítica (incluyendo niveles de vitamina B12), una RM cervical y un estudio neurofisiológico.

La RM cervical (Magnetom-Trio, Siemens, Erlangen, Germany) mostró en secuencias potenciadas en T2 en el plano sagital una hiperintensidad de señal sin efecto de masa a nivel cordonal posterior que se extendía desde C1-C2 a C6-C7 (Fig. 1A). En los cortes axiales y coronales se apreció que la afectación era bilateral y simétrica (Fig. 1BC). Estos hallazgos son característicos de la DCS. No se observaron signos de polineuropatía en el estudio de velocidades de conducción, mientras que los potenciales evocados somatosensoriales estaban desestructurados y con aumento de las latencias en los cuatro miembros, indicando una afectación cordonal posterior.

El hemograma mostró una discreta anemia megaloblástica, con 4,44 10E $12 / \mathrm{L}$ de hematíes (4,7-5,1 10E $\left.{ }^{12} / \mathrm{L}\right), \mathrm{VCM}: 108 \mathrm{fL}(80-95 \mathrm{fL})$ y HCM: 34,9 pg (27-31 pg), siendo la hemoglobina (15,5 $\mathrm{g} / \mathrm{dl})$ y el hematocrito (47,9\%) normales. Los niveles de vitamina B12 en suero fueron de $78 \mathrm{pg} /$ $\mathrm{mL}$ (210-930 pg/mL) y los de ácido fólico de 26,5 $\mathrm{ng} / \mathrm{mL}(2,2-16,9 \mathrm{ng} / \mathrm{mL})$, siendo el resto de las determinaciones bioquímicas en sangre normales. Los anticuerpos frente a células parietales gástricas fueron positivos (1/40), así como los anticuerpos anti-FI (factor intrínseco) (>97,9; normal: 0-25). La biopsia gástrica mostró signos de gastritis crónica de aspecto atrófico, con un aumento del infiltrado inflamatorio.

Con el diagnóstico de DSC el paciente fue tratado con cianocobalamina $(1.000 \mu$ /día por vía intramuscular, durante dos semanas y después mensualmente). En los meses siguientes el paciente presentó una progresiva mejoría de las alteraciones sensitivas. A los 10 meses de evolución, el paciente refería una leve e inconstante sensación de "hormigueos" en los pies y la exploración neurológica solo mostraba una discreta hipopalestesia maleolar. La analítica de control mostró una resolución de la anemia megaloblásica así como de los niveles de vitamina B12 (438 pg/mL). Una RM medular de control, utilizando el mismo equipo, puso de manifiesto la completa desaparición de las lesiones y la normalidad de la intensidad de señal del cordón medular (Fig. 2). 


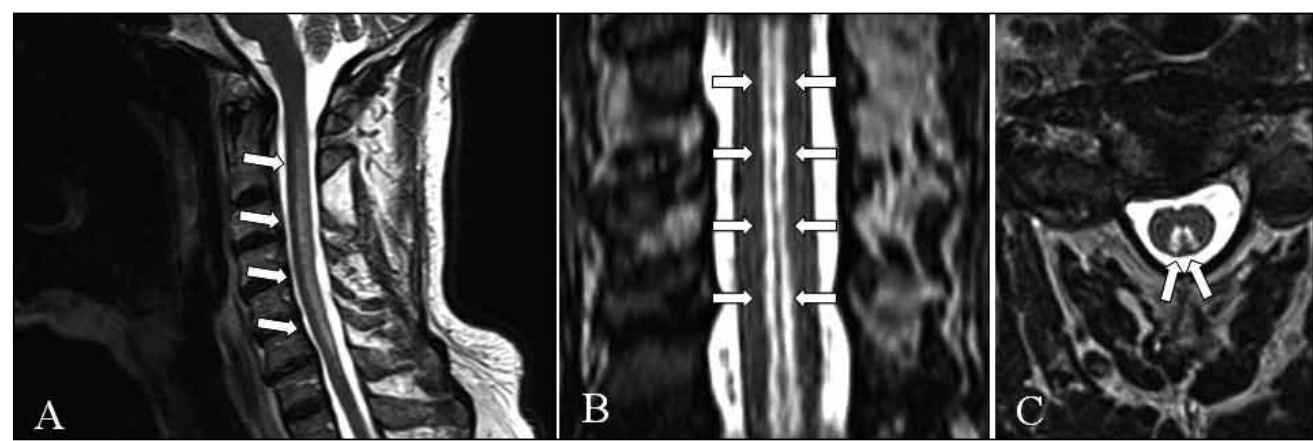

Figura 1. Resonancia magnética del cordón medular a nivel cervical con secuencias potenciadas en T2-TSE realizada 10 meses después del inicio del tratamiento, en la que se observa la resolución completa de los hallazgos típicos de degeneración combinada subaguda. A: corte sagital. B: Reconstrucción coronal. C: Corte axial.
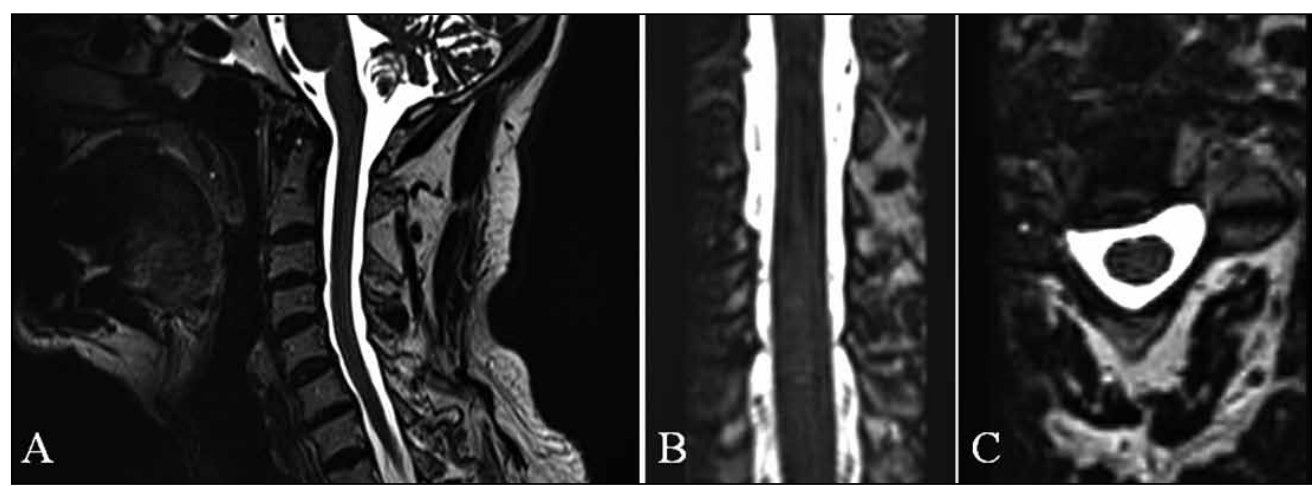

Figura 2. Resonancia magnética del cordón medular a nivel cervical realizada con secuencias potenciadas en T2-Turbo Spin Echo (T2-TSE). A: corte sagital donde se observa una hiperintensidad de señal difusa en la vertiente posterior de la médula espinal, que se extiende desde $\mathrm{C} 1$ hasta $\mathrm{C} 7 \mathrm{sin}$ edema ni efecto de masa (flechas). B: Reconstrucción coronal que muestra una afectación de los cordones posteriores bilateral y simétrica (flechas). C: Corte axial del cordón medular donde se aprecia la afectación cordonal posterior de la médula espinal bilateral y simétrica (flechas).

\section{DISCUSIÓN}

El déficit de vitamina B12 puede cursar con diversas manifestaciones hematológicas, gastrointestinales y neurológicas. La principal manifestación neurológica del déficit de vitamina B12 y con cierta frecuencia la única, es la DCS.

La causa más frecuente de déficit de vitamina $\mathrm{B} 12$ es la anemia perniciosa, una enfermedad autoinmune en la que se producen anticuerpos contra el FI, y que se caracteriza por una gastritis atrófica tipo A con pérdida de células parietales, que producen el FI indispensable para la absorción de la cobalamina. Otras causas de déficit de vitamina B12 son por malabsorción intestinal y/o gástrica, carencias nutricionales o por fármacos (colchicina, metformina $)^{5}$.

Las primeras manifestaciones clínicas de la DCS son las parestesias en forma de "hormigueos" en manos y pies pudiendo llegar a ser incluso normal la exploración neurológica. En las fases más evolucionadas puede haber ataxia sensorial e inesta- 
bilidad e incluso debilidad progresiva con signo de Babinski bilateral por la afectación piramidal. En el caso que se presenta, el trastorno de sensibilidad, por su distribución en guante y calcetín, puede aparentar el de una neuropatía, pero cuando además existe una alteración de la sensibilidad vibratoria con preservación de reflejos es obligado considerar en primer lugar una lesión medular con afectación de los cordones posteriores. La normalidad de las velocidades de conducción junto con las alteraciones en RM medular y en los potenciales evocados demuestran que la sintomatología se debía a la afectación cordonal posterior inducida por un déficit de vitamina B12. La escasa especificidad de los síntomas al inicio dificultan el diagnóstico, y de hecho se ha estimado que desde que se inician las primeras manifestaciones clínicas hasta que se establece el diagnóstico de DCS transcurren alrededor de 4 meses $^{6}$. La importancia del diagnóstico precoz radica en que la rapidez en el inicio del tratamiento condicionará el grado de recuperación de los déficits neurológicos. Si los pacientes con DCS no se tratan precozmente, tanto los síntomas como las lesiones en RM pueden ser irreversibles, causando una atrofia medular y déficits neurológicos permanentes ${ }^{6,7}$.

En la RM medular de los pacientes con DCS, como en el presente caso, se observa la presencia de una hiperintensidad de señal bilateral y simétrica en los cordones posteriores en secuencias potenciadas en $\mathrm{T} 2$, sobre todo en región cervical y dorsal alta, que también puede afectar con menor frecuencia a los cordones anteriores y laterales $^{2-4}$. Desde el punto de vista de imagen de resonancia, la extensión, localización y sobre todo la simetría de las alteraciones de señal medulares son muy características de la DCS, y la administración de contraste paramagnético no se realiza de forma rutinaria puesto que no aporta información adicional ${ }^{6}$. En el diagnóstico diferencial de lesiones medulares con afectación cordonal posterior podrían incluirse las formas espinales puras de esclerosis múltiple, mielitis infecciosas, infarto espinal, o déficit de cobre entre otras ${ }^{8,9}$. Por otro lado, como se demuestra en este caso, la RM puede ser también una buena técnica para monitorizar la respuesta al tratamiento, al encontrarse una correlación entre la disminución de la hiperseñal medular y la mejoría clínica ${ }^{3}$. Se ha observado que habitualmente la normalización de la señal medular en RM precede a la mejoría clínica ${ }^{10}$.

En conclusión, la identificación de una hiperseñal cordonal posterior y simétrica a nivel cervical en la RM, es una hallazgo característico de la DCS por déficit de B12 que orienta el diagnóstico clínico en fases iniciales. La resolución de las alteraciones de RM, precede a la mejoría clínica, y permite monitorizar la eficacia del tratamiento médico.

\section{BIBLIOGRAFÍA}

1. Scalabrino G. Cobalamin (vitamin B12) in subacute combined degeneration and beyond: traditional interpretations and novel theories. Exp Neurol 2005; 192: 463-479.

2. Karantanas AH, Markonis A, Bisbiyiannis G. Subacute combined degeneration of the spinal cord with involvement of the anterior columns: a new MRI finding. Neuroradiology 2000; 42: 115-117.

3. Rabhi S, MaAroufi M, Khibri H, Belahsen F, Tizniti F, BERRADY R ET AL. Magnetic resonance imaging findings within the posterior and lateral columns of the spinal cord extended from the medulla oblongata to the thoracic spine in a woman with subacute combined degeneration without hematologic disorders: a case report and review of the literature. J Med Case Reports 2011; 5: 166-169.

4. Timms SR, CurE JK, KurEnt JE. Subacute combined degeneration of the spinal cord: MR findings. AJNR Am J Neuroradiol 1993; 14: 1224-1227.

5. Yamada K, Shrier DA, TanaKa H, Numaguchi Y. A case of subacute combined degeneration: MRI findings. Neuroradiology 1998; 40: 398400.

6. Bassi SS, Bulundwe KK, GreefF GP, Labuscagne JH, GLEDHILL RF. MRI of the spinal cord in myelopathy complicating vitamin B12 deficiency: two additional cases and a review of the literature. Neuroradiology 1999; 41: 271274. 
7. Bou-Haidar P, Peduto AJ, Karunaratne N. Differential diagnosis of $\mathrm{T} 2$ hyperintense spinal cord lesions: Part B. J Med Imaging Radiat Oncol 2009; 53: 152-159.

8. Maamar M, Mezalek ZT, Harmouche H, Adnaoui, Aouni M, MaAouni A. Contribution of spinal MRI for unsuspected cobalamin deficiency in isolated sub-acute combined degeneration. Eur J Intern Med 2008; 19: 143-145.
9. Misra UK, Kalita J. Comparison of clinical and electrodiagnostic features in B12 deficiency neurological syndromes with and without antiparietal cell antibodies. Postgrad Med J 2007; 83: 124-127.

10. Berlit P, Ringelstein A, Liebig T. Spinal MRI precedes clinical improvement in subacute combined degeneration with B12 deficiency. Neurology 2004; 63: 592. 
